\title{
Disclosure Dilemmas: Ethics of Genetic Prognosis after the 'Right to Know/Not to Know' Debate Christoph Rehmann-Sutter \& Hansjakob Muller (eds.) Ashgate 2009
}

\section{DANIELE CARRIERI ${ }^{1}$}

This book aims to enrich current debates on the ethics of disclosing genetic information. It comprises a collection of essays written by contributors with a range of disciplinary backgrounds: bioethics, genetic medicine, medical anthropology, philosophy, law and sociology, to cite a few. By adopting an interdisciplinary approach, the volume advocates for a theoretical and methodological extension of the ethics of biomedicine.

This interdisciplinary approach allows the volume to add key contextual elements to the classic ethical discussions of the right to know/not to know. It highlights in particular the importance of exploring patients' and families' perspectives on genetic information and their experiences with the healthcare system.

While standard bioethical thinking on genetic medicine and disclosure has tended to focus on the clinical setting and healthcare professionals' disclosure dilemmas, this volume strongly emphasises that patients and families are active agents who are not mere objects of these dilemmas, but constitutive subjects.

Moreover, the content of disclosure - i.e. genetic information itself - cannot be reduced to biomedical interpretative models, but it is rather the result of a more complex and dynamic process of negotiation which involves genetic service providers and patients. Ethical reflection on biomedicine should consider both ends of communication in the patient-professional relationship.

The importance of a constructive integration of local practical realities of individual cases and abstract ethical thinking is stressed throughout the volume. Universal ethical principles and the complex particular realities of clinical cases are presented as being equally crucial and standing in a close and mutual relationship. In this volume, the empirical and theoretical levels of research are strongly intertwined and inform each other, drawing a picture of a 'fluid' ethics (as opposed to a 'fixed' and excessively abstract one).

Clinical cases and empirical research do not provide a model to answer ethical questions, but play a fundamental role in identifying and highlighting the pressing problems and needs of patients and the other actors who are involved in these disclosure practices. Ethical thinking alone cannot identify these problems, but, at the same time, ethical norms provide necessary guiding principles that help to address individual cases. 
Having said that, I sensed some tension between this overall message emphasised in the introduction and conclusion of the book, and the more 'extreme' views expressed in some chapters (e.g. Chapter 2 ) which advocated the priority of sociological investigation of local realities of patients and families over ethical research.

\section{Structure and comments}

The book is composed of 18 chapters (introduction and conclusion excluded) grouped in three parts. The first part titled 'Setting the Scene' introduces the reader to the philosophical, sociological and medical reflections which constitute the leitmotif of the whole volume.

Although the last decade has seen considerable innovations in genetic medicine, the right to know/not to know framework is still viewed as a fundamental heuristic tool to understand the ethical issues arising from genetic testing and screening. However, another key point made in this section is the importance of integrating this framework with a scrupulous understanding of patients' social contexts. The healthcare system and more specifically the clinic - is a crucial part of this context. From the perspective of genetic service providers, there can often be tensions between the professional and deontological drive to reach high ethical standards and the practical functional problems related to the lack of healthcare infrastructures that allow for these standards to be met.

The second part, 'Cases and Issues', reflects the theoretical and methodological guiding principles of the book, presenting a collection of studies which combine clinical cases, qualitative research with patients and ethical and legal analysis.

The importance of family dynamics seems to be one of the most prevalent themes among the many tackled in the seven chapters that compose this section. Given the familial implications of genetic knowledge, family members are key actors in the process of disclosure. Genetic service providers can use the family as a resource when they are dealing with complex cases of disclosure (e.g. unsolicited disclosure about a genetic disease to other relatives). Family relations can shape patients' experiences and understanding of genetic knowledge, strongly influencing their decision-making processes. An awareness of the familial context allows genetic service providers to offer a better service and to manage complex issues arising in clinical practice. Therefore, the familial dimension, or an 'ethics of kinship' (Chapter 5) should complement bioethical discussions and research on genetic medicine.

An interesting contrast is also highlighted between the work of the clinic which is - or should be - grounded in the local realities of familial clinical cases and the general rules and statements of principle offered by the legal system. The court relies on fixed (often 'extreme') cases that may not dovetail with local families' situations and conflicts.

Other chapters in this section question the practical utility and relevance of many susceptibilities tests which are currently offered to patients (e.g. the APOE gene test for Alzheimer disease). Genetic information is probabilistic and can be difficult for 
patients and even genetic service providers to interpret. The idea that genes are stable units which have a clear causal link to disease and other human traits has been firmly refuted in the scientific milieu. However, genetic determinism is still vivid in popular 'gene talk', and influences patients' (pre)understanding of genetic information. Thus, complex probabilistic risk estimations of susceptibilities for future disease may be misinterpreted by patients, causing unnecessary anxiety and harm or offering unjustified reassurances. Policy makers should take this problem into account and consider as well that people are likely to undergo testing as they may be pressurised by the 'technological imperative', as well as by the drive to minimise risk that characterise our society.

The third part, 'Responsibilities', is the most heterogeneous. Overall, it deals with legal, moral and ethical responsibility in communicating genetic information in clinical settings and within family networks.

In this section, a model of 'ethics of communication' is delineated. This model is based on a sense of reciprocity between genetic service providers and patients. In the clinical encounter, it is not only the patient who may present a 'knowledge deficit'. Genetic service providers may rely excessively on their biomedical epistemic standpoints, which can hinder their ability to assess patients' actual understanding of genetic information. Genetic service providers should stress the multifactorial and probabilistic nature of genetic information in order to balance and clarify common over-expectations of testing (fomented by deterministic 'gene talk'). The importance of translating statistical figures based on population databases into 'individualised information giving' which is meaningful for the patient is also emphasised. Ongoing dialogue and collaboration between professionals and patients helps to clarify what genetic information means for the patients. This may also ease some dilemmas arising from clinical practice. In sum, the goal of this ethics of communication is to achieve a good and functional patient-professional rapport which is seen as the key for the promotion of patients' autonomy and, ultimately, wellbeing.

Another interesting link between this and the second part of the book is the exploration of genetic service providers' responsibility to disclose genetic information to the relatives of the index patient, in other words, how far genetic service providers' duty of care should extend. Although the importance of adopting a case-by-case approach is emphasised, it is also suggested that when there is a serious risk for relatives, difficulties in communications with patients and families should not be a justification for inaction. However, I would argue that assessing the degree of severity of risk that legitimises disclosure can also be a difficult task.

The third part explores a gamut of other disparate themes, such as the differences in the legal systems across countries, differences which are reflected in different legal consequences in cases of breach of professionals' duty of care, and the influence that socio-political agendas, whether overt or hidden, can exert on patients' decisionmaking processes in relation to genetic testing and screening. 
Overall, this book sketches a thorough map of some of the most pressing ethical issues arising from current developments in biomedicine. Its attempt to bridge the gaps between bioethics, genetic medicine, law and sociology is very valuable and makes the volume potentially useful to those from any of these disciplines who are approaching the subject. In particular, this volume shows quite compellingly the importance of taking seriously into account the medical, legal and social contexts in which biomedicine is situated. It will certainly enrich those who are interested in the applied ethics of biomedicine.

${ }^{1}$ Egenis ESRC Centre for Genomics in Society, University of Exeter. dc233@exeter.ac.uk 